\title{
OCORRÊNCIA DO ÁCARO BRANCO Polyphagotarsonemus latus (Banks) EM JAMBU Spilanthes oleracea E OUTRAS PLANTAS HOSPEDEIRAS NO ESTADO DO AMAZONAS
}

\author{
Geraldo José Nascimento de Vasconcelos ${ }^{1}$; Sabrina Cruz de Lima²; Neliton Marques da \\ Silva ${ }^{3}$. \\ ${ }^{1}$ Professor Doutor da Universidade do Estado do Amazonas (UEA), Centro de Ensino Superior de Itacoatiara, \\ Itacoatiara, Amazonas, Brasil, gjnvasconcelos@yahoo.com.br \\ ${ }^{2}$ Graduanda do curso de Agronomia da Faculdade de Ciências Agrárias, UEA, Manaus, Amazonas, Brasil, \\ lima.sabrina@ymail.com \\ ${ }^{3}$ Professor Doutor da Faculdade de Ciências Agrárias, UEA, Manaus, Amazonas, Brasil, nmerinato@gmail.com
}

RESUMO: O jambu, Spilanthes oleraceae, é uma hortaliça não convencional nativa da Amazônia, amplamente cultivada como hortaliça folhosa em vários municípios da região Norte. Poucas pragas têm sido constatadas e efetivamente identificadas nesta cultura, não havendo nenhum registro de ácaro fitófago. Assim, o objetivo deste trabalho foi registrar pela primeira vez a ocorrência de Polyphagotarsonemus latus (Banks) em cultivos de jambu, caracterizando os danos e relatar a ocorrência deste ácaro em outras plantas hospedeiras no Estado do Amazonas. Os principais danos causados por $P$. latus em folha de jambu são o enrijecimento e encarquilhamento das folhas jovens, deixando-as impróprias para comercialização.

PALAVRAS-CHAVE: ácaros praga, agricultura tropical, diversidade.

\section{OCCURRENCE OF THE BROAD MITE Polyphagotarsonemus latus (Banks) IN Spilanthes oleracea AND OTHER HOST PLANTS IN AMAZONAS STATES, BRAZIL}

ABSTRACT: Spilanthes oleracea is an unconventional vegetable native to the Amazon, is widely cultivated in several municipalities of the North Brazil. Few pests have been found and identified effectively in this culture, with no record to phytophagous mite. Thus, the purpose of this study was to record the first occurrence of Polyphagotarsonemus latus (Banks) in S. oleracea crops, characterizing the damage and reporting the occurrence of other host plants in Central Amazonia. The main damage caused by $P$. latus in leaf of $S$. oleracea are hardening and shrinking of the younger leaves, leaving them unfit for marketing.

KEY WORDS: diversity, pest mites, tropical agriculture.

O jambu, Spilanthes oleraceae, é uma hortaliça não convencional nativa da Amazônia pertencente à Família Asteraceae (CARDOSO; GARCIA, 1997). Seu cultivo é amplamente difundido, como hortaliça folhosa, por pequenos produtores em vários municípios da região Norte do país, sobretudo nos estados do Amazonas e Pará. É muito utilizada na culinária local como um dos principais ingredientes de iguarias regionais como o tacacá e o pato no tucupi. Além da utilização na alimentação cotidiana, suas folhas também são empregadas na medicina popular para o tratamento da dispepsia, 
malária, infecções da boca e garganta, contra a avitaminose C, como antibiótico e anestésico, sendo estas propriedades atribuídas ao óleo essencial spilanthol, produzido nas folhas mais tenras (COUTINHO et al., 2006).

Poucos organismos-praga tem sido constatadas e efetivamente identificadas nesta hortaliça, tais como paquinha (Gryllotalpidae), grilo (Gryllidae), lagartarosca (Noctuidae) e pulgão (Aphidae), porém a ocorrência é sempre esporádica, não havendo nenhum registro de ácaro fitófago sobre esta cultura.

O ácaro branco, Polyphagotarsonemus latus (Banks), é uma das três espécies de ácaro fitófago mais importante para o Brasil (MORAES; FLECHTMANN, 2008). Este ácaro é uma praga cosmopolita, ocorrendo em países das regiões tropicais e temperadas quentes, mas também pode ser encontrado em casas de vegetação em regiões de clima frio (LINDQUIST， 1986; GERSON， 1992). Apresentando baixa especificidade em relação ao hospedeiro, este ácaro ocorre em mais de 60 famílias de plantas entre dicotiledôneas, monocotiledôneas e gimnospermas (GERSON, 1992). É relatado mais comumente em plantas cultivadas, porém também pode ser encontrado em plantas invasoras como Bidens sp., Galinsoga sp. e Datura sp. (LAVOIPIERRE, 1940), ou até mesmo em Cannabis sativa (GOFF, 1987). É favorecido por condição climática quente e úmida, podendo ter várias gerações em poucas semanas. Estas condições ocorrem durante todo o ano no Estado do Amazonas. Devido à sua frequente e severidade, ocasiona redução da produtividade e aumento do custo de produção, por conta da necessidade de pulverizações constantes de Agrotóxicos, sendo considerado praga-chave em diferentes culturas.

No Brasil P. latus já foi registrado por diferentes autores em vária regiões e culturas, como na Bahia sobre Datura stramonium, Malpighia sobrearginata, Phaseolus vulgaris e Vitis sp., Distrito Federal sobre Capsicum annuum, Capsicum baccatum e Capsicum frutescens, Mato Grosso sobre Hevea brasiliensis, Minas Gerais sobre $C$. frutescens, Glycine max e H. brasiliensis, Paraná sobre Gossypium herbaceum e Ipomoea cairica, Pernambuco sobre $C$. annuum, Carica papaya, D. stramonium, M. sobrearginata, P. vulgaris e Vitis sp., Rio de Janeiro sobre $C$. annuum e $C$. frutescens, Rio Grande do Sul sobre G. max, Ilex paraguariensis e Vitis sp., Roraima sobre $C$. annuum, Santa Catarina sobre Coffea sp. e $H$. brasiliensis, São Paulo sobre Acnistus cauliflorum, C. annuиm, C. papaya, Chamaesyce hirta, Citrus limon, Citrus sinensis, Coffea canephora, Euphorbia heterophylla, G. hirsutum, H. brasiliensis, $P$. vulgaris, Solanum tuberosum e Vitis vinifera 
e Tocantins sobre Jatropha curcas (SARMENTO et al., 2011). Além dos hospedeiros já mencionados, Flechtmann, (1967; 1977) e Moraes e Flechtmann (2008) também relatam a ocorrência do ácaro branco sobre Begonia sp., Cosmos sp., Cydonia oblonga, Dahlia pinnata, Hydrangea macrophylla, Impatiens walleriana, Passiflora spp., Persea americana, Pyrus communis, Pyrus malus, Saintpaulia ionantha, Solanum lycopersicum e Solanum melongena, sem mencionar a procedência.

O objetivo deste trabalho foi registrar a ocorrência do ácaro-branco em cultivos de jambu, caracterizando os danos causados a cultura e relatar a ocorrência deste ácaro em outras plantas hospedeiras na Amazônia Central.

As coletas foram realizadas entre agosto de 2006 e julho de 2010, em áreas de produção localizadas nos municípios de Manaus e Iranduba. Foram coletas amostras de folhas apicais, ramos e frutos, quando presentes, nas seguintes planta Abelmoschus esculentus (Quiabo), C. annuum (pimentão), Capsicum chinense (pimenta-de-cheiro), Jatropha sp. (pinhão manco), S. lycopersicum (tomateiro), S. oleracea, Turnera sp. (chanana), V. unguiculata (feijão de praia) e V. unguiculata cv.gr. sesquipedalis (feijão de metro). O material coletado foi colocado em saco plástico etiquetado e acondicionado em caixa de isopor com gelo. Em seguida foi encaminhado para o laboratório onde os ácaros foram quantificados com o auxílio de estereomicroscópio. Alguns exemplares dos ácaros de cada cultura foram montados em lâmina com meio de Hoyer para microscopia. Após as montagens estes foram identificados em microscópio com contraste de fases, com auxílio do trabalho de Jeppson et al. (1975). Espécimes voucher foram depositados na coleção do Laboratório de Entomologia e Acarologia Agrícola da Universidade Federal do Amazonas e estão disponíveis para consulta, "in loco", e empréstimo interinstitucional.

Após a identificação, foi confirmada a ocorrência de $P$. latus em todas as amostras (Tabela 1). Quando vivos, os machos e fêmeas desta espécie são translúcidos. Devido a sua coloração e tamanho reduzido são difíceis de visualizar a olho nu. Encontra-se usualmente nas partes mais tenras da planta, alimentando-se das jovens folhas apicais, ou frutos novos. As injúrias são causadas nas células mais superficiais da epiderme, devido ao curto estilete desse tarsonemídeo, com cerca de 40 micrometros (JEPPSON et al., 1975). De forma geral, quando ocorre em folhas, estas ficam torcidas, mais rígidas e com margem ondulada (BASSETT, 1981). Nos frutos podem causar manchas necróticas e rachaduras (CROSS; BASSETT, 1982). Mesmo após a eliminação dos ácaros, as folhas jovens continuam, por alguns dias, 
apresentando os danos mencionados, sugerindo que estes possam ser proporcionados por toxinas injetadas pelo ácaro ao alimentar-se (DHOORIA; BINDRA, 1977).

Dos nove hospedeiros registrados, sete são cultivados comercialmente na região de Manaus. Dois novos hospedeiros foram registrados para $P$. latus, $S$. oleraceae e Turnera sp. O primeiro foi constatada uma quantidade média de $32 \pm 11$ ácaros (larva+"pupa"+adulto) por ramo. Nesta cultura as folhas infestadas ficam rígidas $\mathrm{e}$ com as bordas recurvadas para baixo, dando um aspecto de encarquilhamento, a coloração na superfície adaxial fica verde brilhante e na superfície abaxial surgem manchas de coloração bronzeada. As folhas assim atacadas ficam pequenas e com aparência imprópria para a comercialização.

Tabela 1. Planta hospedeira, local e data de coleta e espécimes de P. latus examinado por hospedeiro.

\begin{tabular}{|c|c|c|c|}
\hline Hospedeiro & Local & Coleta & Ácaros identificados \\
\hline Abelmoschus esculentus & Manaus & set./2009 & 90 e 28 q \\
\hline Cарsicum аппиит & Iranduba & $\begin{array}{l}\text { ago./2006 } \\
\text { mar./2008 }\end{array}$ & $\begin{array}{c}11 \text { o e } 24 \text { + } \\
4 \text { त e } 32 \text { o }\end{array}$ \\
\hline & Manaus & $\begin{array}{l}\text { jul./2008 } \\
\text { set./2009 }\end{array}$ & 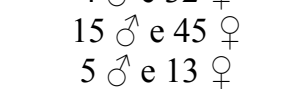 \\
\hline Capsicum chinense & Iranduba & $\begin{array}{l}\text { ago./2006 } \\
\text { mar./2008 }\end{array}$ & $\begin{array}{c}20 \text { e } 8 \text { क } \\
60 \text { e } 19+9\end{array}$ \\
\hline Jatropha sp. & Manaus & mar./2010 & 12 ○े e 30 क \\
\hline Solanum lycopersicum & Manaus & set./2009 & 13 ô e 53 o \\
\hline Spilanthes oleracea & Manaus & jul./-2010 & $8 \mathrm{O}^{\pi} \mathrm{e} 42$ ? \\
\hline Turnera sp. & Manaus & fev./2010 & 11 우 \\
\hline Vigna unguiculata & Manaus & nov./2009 & 15 o e 38 q \\
\hline Vigna unguiculata $\mathrm{cv} . \mathrm{gr}$. sesquipedalis & Manaus & jul./2010 & 60 e 21 ? \\
\hline
\end{tabular}

Embora não haja nenhum produto registrado para o controle do ácaro branco em jambu, o mesmo está sendo feito através de aplicações de acaricida a base de Abamectina na dose de $30 \mathrm{~mL}$ do produto comercial para 100 L de água, com 15 dias de carência.

Em Turnera sp., P. latus foi encontrado na superfície abaxial de folhas apicais. Nesta superfície foram observadas manchas bronzeadas, semelhantes as causadas por $P$. latus em outras culturas. A ocorrência deste ácaro em Turnera sp. é um relato importante, pois trata-se de uma planta daninha com ampla distribuição encontradas em vários agroecossistemas, podendo servir como hospedeiro alternativo para $P$. latus, possibilitando a permanência desta praga na área de produção, mesmo na ausência de plantas cultivadas suscetíveis. 


\section{CONCLUSÕES}

Devido às condições climáticas favoráveis a $P$. latus no Amazonas e ao aumento do cultivo de jambu em condições semi-protegida, é provável que este ácaro se alastre na região, atingindo o status de pragachave para a cultura e exigindo novas táticas de controle.

\section{REFERÊNCIAS}

BASSETT, P. Observations on broad mite (Polyphagotarsonemus latus) (Acari: Tarsonemidae) attacking cucumber. Crop Protection, v. 1, p. 99-103, 1981.

CARDOSO, M. O.; GARCIA, L. C. Jambu (Spilanthes oleracea L.). In: CARDOSO, M.O. (Co.). Hortaliças não-convencionais da Amazônia. Brasília: Embrapa-SPI, 1997; Manaus: Embrapa-CPAA, p.133-140.

COUTINHO, L. N. et al. Galhas e deformações em jambú (Spilanthes oleraceae) causadas por Tecaphora spilanthes (Ustilaginales). Summa Phytopathologica, v. 32, n. 3, p. 283-285, 2006.

CROSS, J. V.; BASSETT, P. Damage to tomato and aubergine by broad mite, Polyphagotarsonemus latus (Banks). Plant Pathology, v. 31, p. 391-393, 1982.
DHOORIA, M. S.; BINDRA, O. S. Polyphagotarsonemus latus (Banks) a mite pest of chilli and potato in Punjab. Acarology Newsletter, v. 4, p. 7-9, 1977.

FLECHTMANN, C. H. W. Ácaros de plantas hortícolas. Boletim Técnico Cientifico/Escola Superior de Agricultura Luiz de Queiroz, v. 29, p. 1-20, 1967.

GERSON, U. Biology and control of the broad mite, Polyphagotarsonemus latus (Banks) (Acari: Tarsonemidae). Experimental and Applied Acarology, v. 13, p. 163-178, 1992.

GOFF, M. L. A catalog of the Hawaiian Islands. University of Hawaii Research Extension Series, v. 75, p. 1-75, 1987.

JEPPSON, L. T. et al. Mites injurious to economic plants. Berkeley: University of California Press, 1975. 614p.

LAVOIPIERRE, M. M. J. Hemitarsonemus latus (Banks) (Acarina), a mite of economic importance new to South Africa. Journal of the Entomological Society of Southern Africa, v. 3, p. 116-123, 1940.

LINDQUIST, E. E. The world genera of Tarsonemidae (Acari: Heterostigmata): a morphological, phylogenetic, and systematic revision, with a reclassification of familygroup taxa in the Heterostigmata. Memoirs of 
the Entomological Society of Canada, v. 136, p. 1-517, 1986.

MORAES, G. J.; FLECHTMANN, C. H. W. Manual de acarologia: Acarologia básica e ácaros de plantas cultivadas no Brasil. Ribeirão Preto: Holos, 2008. 288 p.

SARMENTO, R. A. et al. Suitability of the predatory mites Iphiseiodes zuluagai and Euseius concordis in controlling Polyphagotarsonemus latus and Tetranychus bastosi on Jatropha curcas plants in Brazil. Experimental and Applied Acarology, v. 53, p. 203-214, 2011. 